\title{
MULTIFUNCTIONAL TOOL FOR EXPANDING AFM-BASED APPLICATIONS
}

\author{
S. Deladi, N.R. Tas, G.J.M. Krijnen, M.C. Elwenspoek \\ MESA + Research Institute/University of Twente, Transducers Science \& Technology Department \\ s.deladi@ewi.utwente.nl
}

\begin{abstract}
A multifinctional tool which expands the application field of atomic force microscope-based surface modification is presented. The AFM-probe can be used for surface modification and in-situ characterization at the same time, due to a special configuration with two cantilevers. Various applications from different fields are presented, which were carried out with one and the same tool: in-situ characterization of wear generated with and without local lubrication (tribology), fountain-pen lithography in which material is deposited or removed (physical-chemistry), and electrochemical metal deposition (electro-chemistry).
\end{abstract}

Keywords: fountain pen, AFM, lithography

\section{INTRODUCTION}

The evolution of atomic force microscopy enables novel applications which required different experimental set-ups and special instrumentation in the past. This work presents a multifunctional tool which used in an Atomic force Microscope (AFM) allows a wide range of different applications with the same instrument. Moreover, new surface modification and characterization possibilities are facilitated with a special configuration of the tool.

In this section the state of the art of different applications and the extension or improvement of the techniques by using the multifunctional probe are presented.

State of the art nanoscale wear studies [2] use the same tip for wearing and measuring, wearing is done at a high load while subsequent imaging is done with the same probe on a larger area applying a smaller load. The novelty of nanotribological and reliability-related applications is that surface modification can be monitored in-situ, in one and the same scan-line, hence it is easy to determine the surface modification history. The topography or friction image obtained with the multifunctional probe comprises a modified and an unmodified (reference) region [1].

AFM-based fountain pen lithography is a new application. Fountain pen probes recently developed $[3,4]$ extend the possibilities of dip-pen lithography [5] (creation of patterns by adsorption of a self-assembled monolayer on a gold surface). Generation of patterns by fluid enabled material removal can also be placed in the category of AFM-based fountain pen lithography. State of the art method to perform this application is based on using pulled glass nanopipettes [6].

Reported electrochemical metal depositions are carried out with glass captliaries and by using special instrumentation [7]. The multifunctional tool enables AFM-based electrochemical metal deposition by closing the electrical circuit between the gold layer on top of the probe (anode) and the substrate (cathode), on which metal deposition is intended, via an electrically conductive liquid.

\section{DESIGN AND FABRICATION}

The tool consists of two cantilevers, a detection and a multifunctional one, connected to the same support (Figure 1), one for surface modification, and the other for in-situ detection of topography change. The laser beam can be focused alternatively on either of the cantilevers, depending on the application. A fluidic reservoir is machined into the support which is connected to fluidic channels embedded in the cantilever used for surface modification (Figure 2). An outlet hole at the tip or at the base of the pyramidal tip enables fluid dispensing locally during scanning.

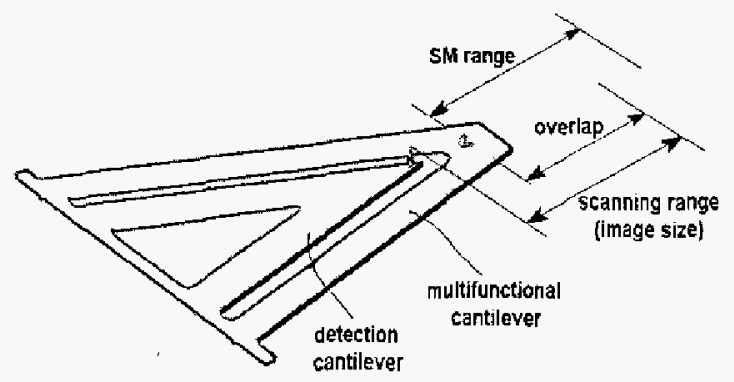

Figure 1. Schematic of the device (bottom side).

The material chosen for the fabrication of the cantilevers is $\mathrm{Si}_{x} \mathrm{~N}_{\mathrm{y}}$ due to its excellent mechanical properties and because its hydrophilic nature facilitates fluid transport in capillaries. Due to good chemical resistance of the $\mathrm{Si}_{\mathrm{x}} \mathrm{N}_{\mathrm{y}}$ to a large variety of chemicals, the application field of the fountain pen probe is hardly limited by the fluids that have to be transported through the channels. The thickness of the cantilevers is chosen in such a way that their elastic properties match those of the commercial ones, in order to allow contact and tapping mode operations with standard AFM equipment. A trapezoidal shape was adopted for the multifunctional

TRANSDUCERS'05

The 13th International Conference on Solid-State Sensors, Actuators and Microsystems, Seoul, Korea, June 5-9, 2005 
cantilevers, in which the fluidic channels are embedded. The multifunctional cantilevers are $200 \mu \mathrm{m}$ long, $15 \mu \mathrm{m}$ wide, and $0.6 \mu \mathrm{m}$ thick, which results in a spring constant of $0.055 \mathrm{~N} / \mathrm{m}$ and a resonance frequency of about $140 \mathrm{kHz}$ for the first vibration mode. The height and the width of the fluidic channels are 0.25 and $2 \mu \mathrm{m}$ respectively. The detection cantilever is V-shaped, like most of the contact mode AFM-probes made of $\mathrm{Si}_{\mathrm{x}} \mathrm{N}_{\mathrm{y}}$. The support of the cantilevers is Pyrex, chosen due to the good $\mathrm{Si} / \mathrm{Pyrex}$ etch selectivity in $\mathrm{KOH}$ solution.

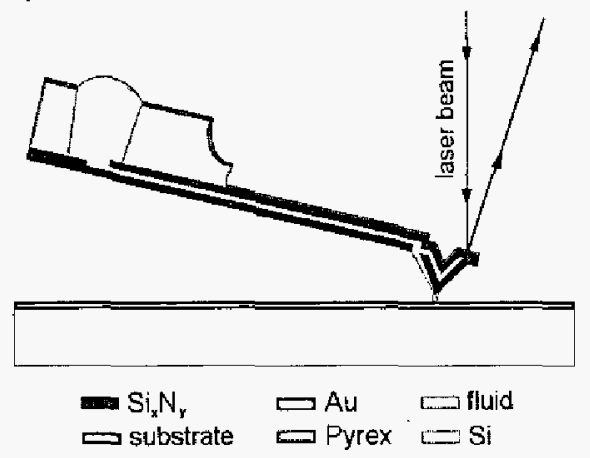

Figure 2. Cross-section of the multifunctional cantilever.

The fabrication process of the device is based on the fabrication of commercial AFM probes combined with techniques for the fluidic functionality. The reservoir of the probe is powder blasted with alumina particles into the Pyrex support of the cantilevers. The fluidic channels are enclosed by two $\mathrm{Si}_{\mathrm{x}} \mathrm{N}_{\mathrm{y}}$ layers and are fabricated by sacrificial layer technique. The fabricated fountain pen probe is presented in Figure 3 .

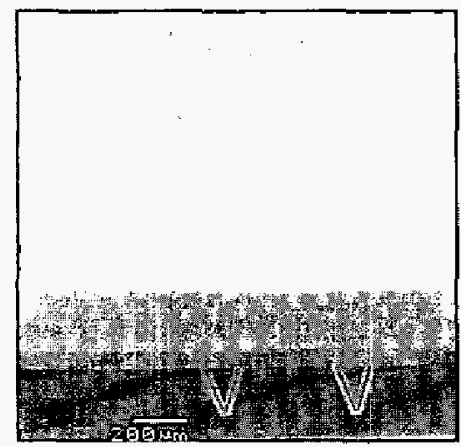

Figure 3. Fabricated fountain pen.

\section{PROOF OF PRINCIPLE}

The fluid transport in the channels was monitored by a CCD camera mounted on an optical microscope. The test was performed before the cantilevers were coated with a gold layer, needed for optical reflection of the laser beam during operation in AFM. The moving meniscus was observed when the reservoir was fed with a water droplet (Figure 4). After coating the probes with a gold. layer, the resonance frequency change of the multifunctional cantilever with empty channels relative to water filled channels was measured in tapping mode AFM. The resonance frequency shift due to additional fluid mass was in agreement with the calculations [4].

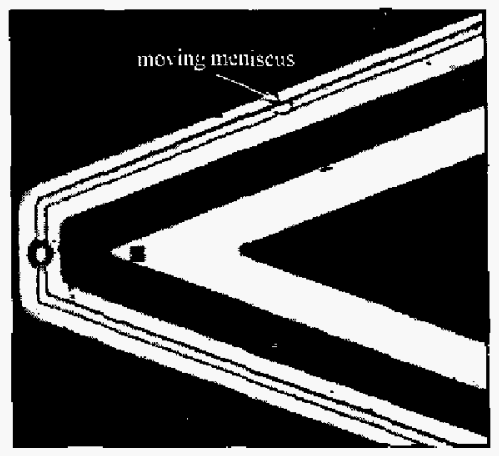

Figure 4. Fluid (water) transport in the microchannel.

The fluid transport from the probe-tip to the substrate was demonstrated by adhesion measurement between the probe-tip and the substrate in dry conditions and with liquid in the reservoir [4]. The dramatic change of the adhesion was the evidence that liquid is transferred to the substrate-tip interface.

\section{APPLICATIONS}

In this section a range of applications is presented which can be carried out by using the developed probes in an AFM.

\section{Tribological applications in dry conditions}

With the configuration presented in Figure 1 it is possible to in-situ monitor the removal of material along a single line as it evolves with the number of scan cycles [1]. When the slow scan axis is disabled and scanning occurs along a single line, the image is built up from topography information of subsequent scan cycles. The multifunctional cantilever of the probe used for this experiment did not contain fluidic channels, which does not alter the conditions of the tests because it is carried out without fluidic contribution.

A Si wafer $\left(R_{\mathrm{a}} \sim 0.4 \mathrm{~nm}\right)$ was chosen as sample, and the test surface on the multifunctional cantilever was square shaped with $5 \mu \mathrm{m}$ sides. The load on the detection tip was optimized so that the surface is not damaged due to scanning with the tip, while the force applied on the edge of the square test surface was $0.4 \mu \mathrm{N}$. In Figure 5 it is shown that the depth of the wear track increases from $0.2 \mathrm{~nm}$ in the first scan to $1.3 \mathrm{~nm}$ for the $256 \mathrm{th}$ scan cycle. The $0.2 \mathrm{~nm}$ deep track after the first scan cycle is attributed to removal of the native oxide layer which forms on the $\mathrm{Si}$ sample in ambient environment. The sample was dipped into a $1 \%$ vol. $\mathrm{HF}: \mathrm{H}_{2} \mathrm{O}$ solution 
before the experiment, so that the native oxide formed on the $\mathrm{Si}$ surface by the inception of the in-situ wear test was thinner than usually.

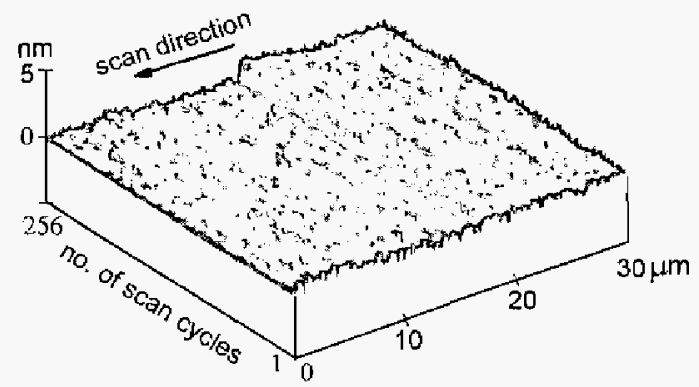

Figure 5. In-situ wear test on a Si surface ( $\operatorname{Ra} \sim 0.4 \mathrm{~nm}$ ). The (trace) image is built up from subsequent scanning along a single line (slow scan axis disabled).

Tribological applications with fluidic contribution

In-situ characterization of fluid enabled surface modification can be carried out when the multifunctional cantilever comprises fluidic channels like in Figurc 2. Rubbing a surface while dispensing liquid can be called chemical-mechanical polishing. The outlet hole of the fluidic channel was focused ion beam milled at the tip of the pyramid located on the multifunctional cantilever.
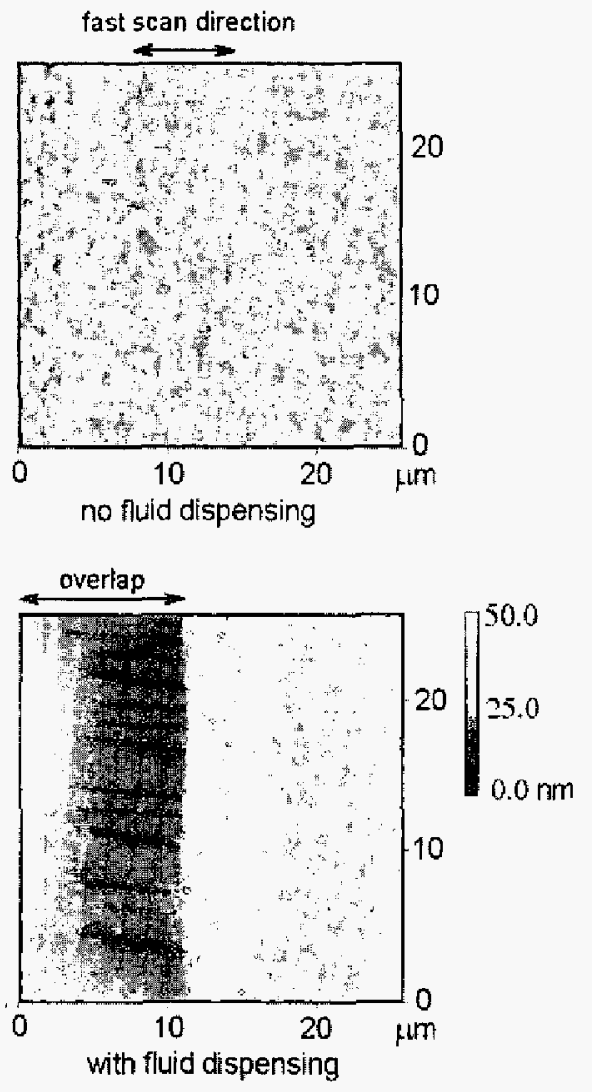

Figure 6. In-situ characterization of fluid enabled surface modification.
A $100 \mathrm{~nm}$ thick sputtered $\mathrm{Cr}$ layer was used as sample surface and the fluid was Chromium etchant Selectipur(B) (MERCK 111547) with ammonium cerium (IV) nitrate as main active component. Frame scanning was performed in dry condition and with fluid dispensed through the multifunctional tip. The scan speed was 80 $\mu \mathrm{m} / \mathrm{s}$, while the contact force on the detection and multifunctional tips were $5 \mathrm{nN}$ and $0.6 \mu \mathrm{N}$ respectively. Figure 6 shows that no surface modification was generated by the multifunctional tip in dry conditions, which demonstrates that the sample surface is hard enough not to be mechanically modified by a $\mathrm{Si}_{x} \mathrm{~N}_{\mathrm{y}}$ tip.

Fluid ( $\mathrm{Cr}$ etchant) was inserted into the reservoir with a syringe and the experiment was repeated. Within the first scan cycle a step of about $16 \mathrm{~nm}$ was detected. In the operating range of the multifunctional tip the liquid dispensed on the sample attacks the $\mathrm{Cr}$ chemically and the tip removes the product mechanically. In the bottom picture of Figure 6 a sharp line which differentiates the regions of modified and unmodified topographies can be observed. The line also represents the end of the scan range of the multifunctional tip.

\section{Fountain pen lithography by material removal}

The same principle, chemical-mechanical topography modification, can be used to create pattems. In this application the features were generated with the pyramidal tip located on the multifunctional cantilever, having the outlet holes opened photolithographically at the base of the pyramid. The detection of the patterns was done by frame scanning with the same tip after it dried. A 25 nm thick chromium layer sputtered on a polished $\langle 100\rangle$ single-crystal $\mathrm{Si}$ substrate was chosen for sample material due to its hardness, thus at a few $\mathrm{nN}$ contact force the sample cannot be scratched mechanically with a $\mathrm{Si}_{x} \mathrm{~N}_{y}$ tip. The fluid used in the test was Chromium etchant Selectipur( $\mathrm{B}$.

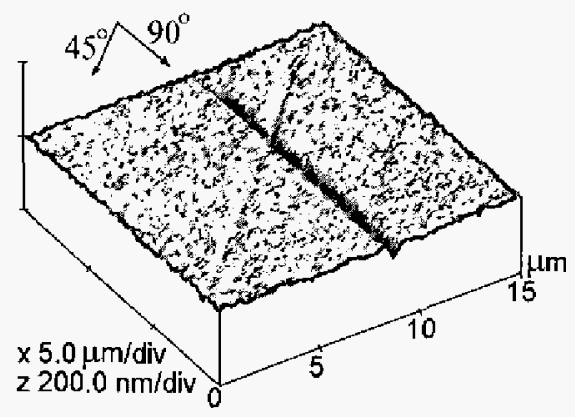

Figure 7. Pattern generated by fluid enabled material removal.

Two lines were etched into the $\mathrm{Cr}$ film, as shown in Figure 7 [4]. A line at $90^{\circ}$ orientation to the longitudinal axis of the cantilever was etched by three scan cycles with $5 \mu \mathrm{m} / \mathrm{s}$ speed at $5 \mathrm{nN}$ contact force, and another at $45^{\circ}$ orientation by just one scan cycle under the same conditions. The first line was $25 \mathrm{~nm}$ deep and $0.8 \mu \mathrm{m}$ wide (the tip reached the Si substrate on which the $\mathrm{Cr}$

TRANSDUCERS'05

The 13th International Conference on Solid-State Sensors, Actuators and Microsystems, Seoul, Korea, June 5-9, 2005 
was sputtered), while the second was $14 \mathrm{~nm}$ deep and $0.35 \mu \mathrm{m}$ wide.

Fountain pen lithography by adsorption of SAM on gold For proof of concept ink consisting of 1 octadecanethiol (ODT) dissolved at $0.1 \mathrm{mM}$ in $\mathrm{CH}_{2} \mathrm{Cl}_{2}$ was selected. The ink forms a Self-Assembled Monolayer (SAM) when brought into contact with a gold surface. The substrate was $20 \mathrm{~nm}$ thick sputtered gold on Si wafer.

Each line in Figure 8 was generated by 5 scan cycles in the direction orthogonal to the longitudinal axis of the cantilever, while the slow scan axis was disabled [4]. The contact force was $1 \mathrm{nN}$ and the scanning speed 4 $\mu \mathrm{m} / \mathrm{s}$.

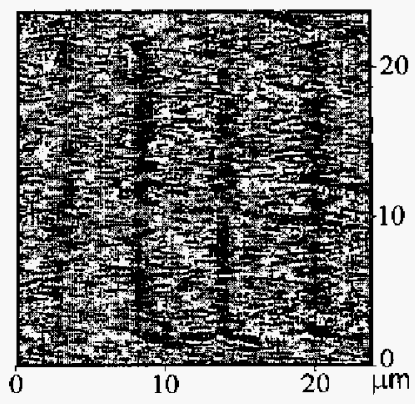

Figure 8. Friction image of the SAM pattern on gold.

The thinnest lines generated by adsorption of SAM on gold surface were $0.5 \mu \mathrm{m}$ wide. We estimate that the width of the lines is determined by the diffusion and wetting due to continuous ink flow, beside the effect of the sharpness of the tip and the roughness of the sample surface. Optimization of the tip geometry, ink flow-rate and smoothness of the sample surface could further decrease the critical width.

\section{Electrochemical metal deposition}

An experiment was carried out to demonstrate that metallic structures can be created by successive deposition of metal layers on a particular region [8]. A $100 \mathrm{~nm}$ thick gold layer sputtered on a $<100>$ singlecrystal Si wafer was used as substrate. Copper structures were created by two subsequent scan cycles on a $1 \times 1$ $\mu \mathrm{m}^{2}$ frame at $0.4 \mu \mathrm{m} / \mathrm{s}$ scan speed. In the experiments $\mathrm{CuSO}_{4}$ dissolved at $1 \mathrm{mM}$ in deionised water was used as electrolyte. The measured conductivity of the electrolyte solution was $176 \mu \mathrm{S} / \mathrm{cm}$, which returns a channel resistance of approximately $110 \mathrm{G} \Omega$ for a $2 \mu \mathrm{m}$ wide, $0.25 \mu \mathrm{m}$ thick and $1 \mathrm{~mm}$ long electrolyte column. The electrochemical deposition was carried out at $10 \mathrm{~V}$ $\mathrm{DC}$, which according to the channel resistance results in $\sim 90$ pA electrical current (calculated).

After scanning the first time, the result was analyzed by frame scanning of a larger area. Deposition of $\mathrm{Cu}$ was observed, as the section analysis in Figure 9 shows. The base of the structure is wider than the initially scanned area, which can be explained by the spreading of the electrolyte solution on the substrate. The height of the structure was $28.1 \mathrm{~nm}$ after the first deposition. After the tip was repositioned on the initial Ix1 $\mu \mathrm{m}^{2}$ frame, a second scanning was carried out, resulting in a hill with a height of $75.3 \mathrm{~nm}$.
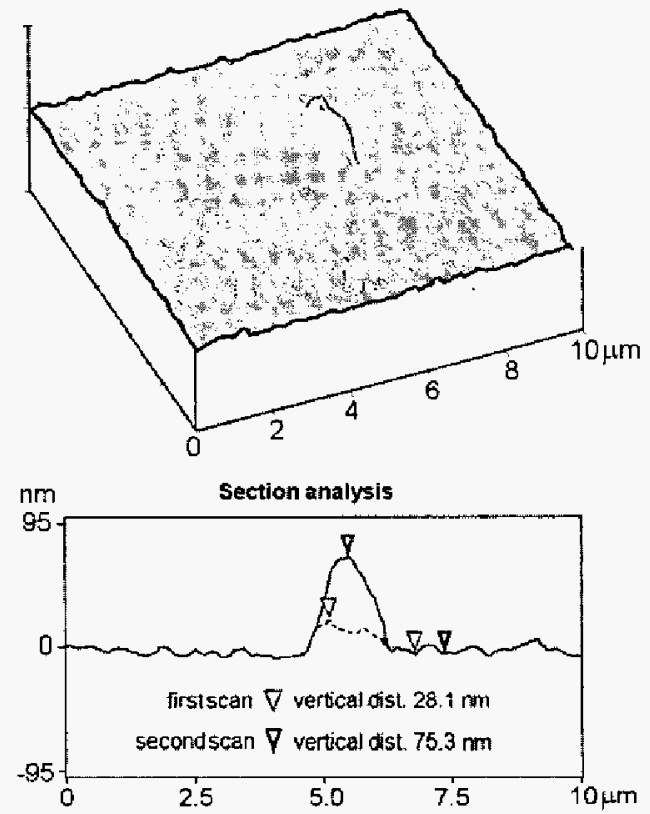

Figure 9. AFM-based electrochemical metal deposition.

\section{CONCLUSIONS}

We have shown the potential of the multifunctional probe by carrying out various applications using standard AFM equipment.

It has been demonstrated that lines down to 0.5 $\mu \mathrm{m}$ widths can be generated by adsoption of SAM on gold or by removing mterial such as chromium. Moreover, tribological applications and electrochemical copper deposition were carried out successfully.

\section{REFERENCES}

[1] S. Deladi et al. Rev. Sci. Instr. 76, pp. 016102 (2005).

[2] B. Bhushan, Wear 251, pp. 1105-23 (2001).

[3] K-H. Kim et al. Proc. of the 4th Int. Symposium on MEMS and Nanotechnology, pp. 235-8 (2003).

[4] S. Deladi et al. Appl. Phys. Lett. 85, pp. 5361-3 (2004).

[5] R.D. Piner et al. Science 283, pp. 661-3 (1999).

[6] A. Lewis et al. Appl. Phys. Lett. 75, pp. 2689-91 (1999).

[7] L. Staemmler et al. Electrochemical and Solid-State Lett. 5, pp. C61-C63 (2002).

[8] S. Deladi et al. Proc. IEEE MEMS'05, pp. 564-7 (2005). 